\title{
A Method for Accurate Amplification of Polymorphic CA-repeat Sequences
}

\author{
Shannon J. Odelberg ${ }^{1}$ and Ray White ${ }^{2}$
}

\footnotetext{
${ }^{1}$ Department of Human Genetics; ${ }^{2}$ Howard Hughes Medical Institute, Department of Human Genetics, University of Utah, Salt Lake City, Utah 84112
}

\begin{abstract}
Anomalous PCR products are often produced during the amplification of $\mathrm{d}(\mathrm{CA})_{\mathrm{n}} \cdot \mathrm{d}(\mathrm{TG})_{\mathrm{n}}$ sequences. Upon denaturing polyacrylamide gel electrophoresis, these products yield a ladder-like pattern that can complicate genotypic interpretation. We have developed two related techniques, referred to as two- and three-stage linear amplification (2-SLA and 3-SLA, respectively), which largely overcome this problem and yield readily interpretable banding patterns.
\end{abstract}

A nomalous products are often generated during the in vitro amplification of $d(C A)_{n} \cdot d(T G)_{n}$ sequences [hereafter referred to as $d(C A)_{n}$ loci, or $d(C A)$-repeats] by PCR. These PCR products usually differ in length by multiples of two nucleotides and form a ladder-like pattern upon denaturing polyacrylamide gel electrophoresis (Fig. 1, lane 1). ${ }^{(1,2)} \mathrm{Be}$ cause this complex pattern can make genotypic interpretation difficult, it is of critical concern when attempting to genotype individuals without the assistance of a known pedigree, for example, for parentage testing or criminal investigations. Even within an established pedigree, these anomalous products can occasionally make it difficult to distinguish between homozygous and heterozygous patterns when the two alleles in question differ by a single dinucleotide repeat.

Approximately 50,000 d(CA) $)_{n}$ loci are present in the human genome; ${ }^{(3,4)}$ an even distribution would place them at $\sim 60$-kb intervals. Most of these loci are polymorphic, and some have heterozygosities $>80 \% .^{(1,5)}$ Their ubiquity and polymorphism make them valuable markers for genetic studies. Thus, it is desirable, if not essential, to develop a technique that will reliably generate interpretable data for this family of genetic markers.

It has been proposed that the anomalous DNA fragments produced during PCR are a result of polymerase slippage during primer extension. ${ }^{(1,2)}$ However, we demonstrate that slippage cannot account for all of the mutant fragments generated during PCR. Alternative mechanisms for the ladder-like pattern include recombinational processes such as "out-of-register" annealing of truncated extension products in subsequent PCR cycles and out-of-register templateswitching during DNA synthesis. Several studies have shown that recombinant molecules can be generated during amplification by PCR; ${ }^{(6-10)}$ however, the recombinational mechanism was not addressed in those studies.

We present two related techniques, referred to as two- and three-stage linear amplification (2-SLA and 3-SLA), which greatly diminish the amount of anomalous amplification products and yield readily interpretable genotypic patterns of $d(C A)_{n}$ loci.

\section{MATERIALS AND METHODS}

\section{Amplification by PCR}

A $10-\mu l$ reaction mixture included $100 \mathrm{fg}$ of the clone pKZ15/228 [contains a $\mathrm{d}(\mathrm{CA})_{25}$ sequence from the LPL1GTR2 marker (S.J. Odelberg, R.B. Weiss, A. Hata, and $\mathrm{R}$. White, in prep.)], $1 \times$ rapid air thermocycler buffer $(50 \mathrm{~mm}$ Tris at $\mathrm{pH} 8.4,3 \mathrm{~mm} \mathrm{MgCl}_{2}, 20 \mathrm{~mm} \mathrm{NaCl}, 500$ $\mu \mathrm{g} / \mathrm{ml}$ of BSA), $500 \mu \mathrm{M}$ of each dNTP, 0.1 pmole of ${ }^{32} \mathrm{P}$-end-labeled primer LP1GT2A (5'-AATGGATGGATAGTTGGGAGAGGG-3'), 4.9 pmoles of unlabeled LP1GT2A, 5 pmoles of RLPdU (5'-CUGGUUCCCACAUGCCCCAUUU- ${ }^{\prime}$ ), and 0.4 unit of AmpliTaq (Perkin-Elmer Cetus, Norwalk, CT). This mixture was drawn into a microcapillary tube by capillary action, and the ends of the tube were flame-sealed. The tube was placed in a 1605 Air Thermo-Cycler (Idaho Technology, Inc., Idaho Falls, ID), and 30 thermal cycles were performed with the following cycling profile: first denaturing phase at $94^{\circ} \mathrm{C}$ for $15 \mathrm{sec}$; subsequent denaturing phases at $94^{\circ} \mathrm{C}$ for $0 \mathrm{sec}$ (temperature spike); annealing phase at 


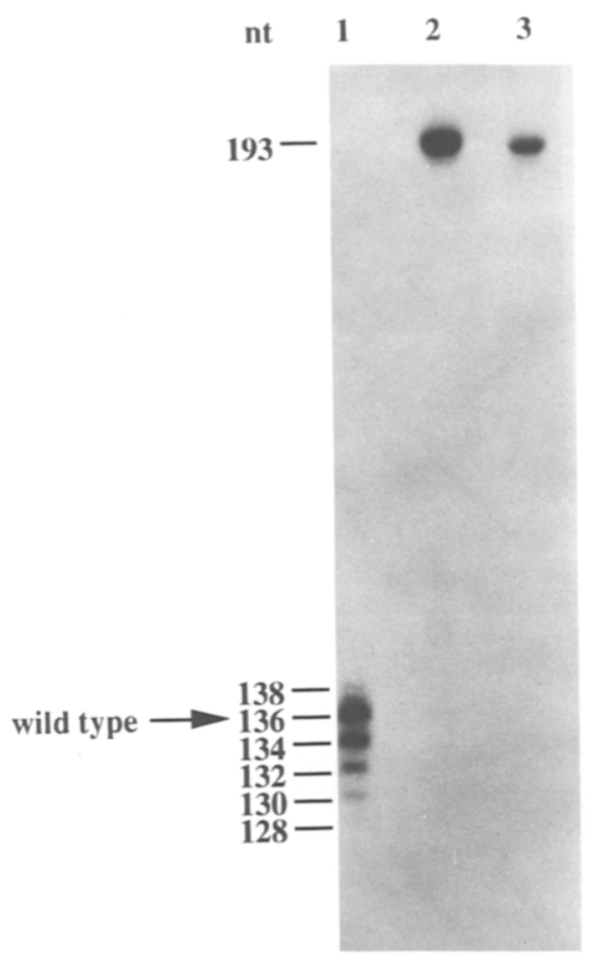

FICURE 1 Comparison of the products generated by PCR, a single round of primer extension, and linear amplification. (Lane 1) PCR products of the LPL1GTR2 marker. The band that represents the wild-type allele is indicated. (Lane 2) A single primer extension through the $\mathrm{d}(\mathrm{CA})$ repeat of the LPL1GTR2 marker to the end of the template. (Lane 3) Linear amplification through the $d(C A)$ repeat of the LPL1GTR2 marker. [Note: Primer extensions and linear amplification through this $d(C A)$ repeat have also been performed using shorter templates (made by cutting the template at a restriction site closer to the repeat); these templates generate extension products that are 78 nucleotides in length and, therefore, can be resolved at single-nucleotide resolution.] Mutation rates obtained from these latter experiments are similar to those obtained from the data shown.

$60^{\circ} \mathrm{C}$ for $0 \mathrm{sec}$ (note that $55^{\circ} \mathrm{C}$ for $0 \mathrm{sec}$ produces indistinguishable results); and extension phase at $74^{\circ} \mathrm{C}$ for $2 \mathrm{sec}$. The ends of the tube were removed with a sapphire cutter, and the sample was expelled into a microcentrifuge tube with the aid of a Captrol III Microdispenser (Drummond, Broomall, PA). Five microliters of loading dye (95\% formamide, 10 $\mathrm{mm} \mathrm{NaOH}, 0.05 \%$ bromphenol blue, $0.05 \%$ xylene cyanol) was added, the sample was denatured at $>90^{\circ} \mathrm{C}$ for 5 min and quenched on ice, and $6 \mu$ l was loaded onto a $7 \%$ denaturing polyacrylamide gel (5.6 м urea, 32\% formamide).
Electrophoresis was performed at a constant 40 watts for $\sim 4 \mathrm{hr}$.

Amplification of human genomic DNA by PCR was identical to the procedure described above, except that $200 \mathrm{ng}$ of initial template DNA was used rather than $100 \mathrm{fg}$, and the annealing phase of the PCR was carried out at $55^{\circ} \mathrm{C}$ for $0 \mathrm{sec}$.

\section{Primer Extension and Linear Amplification}

Clone pKZ15/228 was digested with HaeIII. Approximately $50 \mathrm{ng}$ of pKZ15/ 228-HaeIII was used in a single round of primer extension using 1 pmole of ${ }^{32} \mathrm{P}$ end-labeled LP1GT2A. The buffer and the dNTP and polymerase concentrations were the same as those used for amplification by PCR. The thermal-cycling reaction was performed on the 1605 Air Thermo-Cycler with a 15-sec denaturation phase at $94^{\circ} \mathrm{C}$, a spike at $60^{\circ} \mathrm{C}$, and an extension phase at $74^{\circ} \mathrm{C}$ for $2 \mathrm{sec}$. The sample was prepared for loading in a manner similar to that described for the PCR samples.

The sample used for linear amplification was prepared in the manner described above, except that $\sim 1.7 \mathrm{ng}$ of pKZ15/228-HaeIII was used instead of 50 ng. Thirty thermal cycles were performed using the PCR cycling conditions described in the previous section.

\section{Two- and Three-stage Linear Amplification}

Each $10-\mu$ l reaction contained $200 \mathrm{ng}$ of genomic DNA, $1 \times$ rapid air-cycling buffer (see above, Amplification by PCR), $500 \mu \mathrm{M}$ of each dNTP, 1 pmole of primer LPL1dU (5'-GAAUUCCUGCAGCUCUCAGAUUUUU- $3^{\prime}$ ), and 0.4 unit of AmpliTaq. The samples were subjected to 100 thermal cycles on the 1605 Air Thermo-Cycler with the following profile: initial denaturing phase at $94^{\circ} \mathrm{C}$ for $15 \mathrm{sec}$; subsequent denaturing phases at $94^{\circ} \mathrm{C}$ for $0 \mathrm{sec}$ (temperature spike); annealing phase at $55^{\circ} \mathrm{C}$ for $0 \mathrm{sec}$; and extension phase at $74^{\circ} \mathrm{C}$ for $2 \mathrm{sec}$. Following the linear amplification, 1 unit of uracil-DNA glycosylase (Boehringer Mannheim, Indianapolis, IN) was added to each sample and uracil excision was allowed to occur at $37^{\circ} \mathrm{C}$ for $90 \mathrm{~min}$. The samples were then incubated at $95^{\circ} \mathrm{C}$ for $15 \mathrm{~min}$, extracted once with phenol and once with chloroform, and precipitated with two volumes of absolute ethanol in the presence of $2.5 \mathrm{M}$ ammonium acetate and $2 \mu \mathrm{g}$ of carrier tRNA. The mixture was centrifuged, and the DNA pellet was rinsed once with $70 \%$ ethanol. After drying on a Speed-vac, the samples were resuspended in $10 \mu \mathrm{l}$ of the mixture used in the first linear amplification, except that 1 pmole of primer RLPdU (see above, Amplification by PCR) replaced primer LPL1dU. The samples were subjected to a second round of linear amplification. The cycling profile was identical to the one used in the first round, except that all denaturing steps were performed at $94^{\circ} \mathrm{C}$ for $0 \mathrm{sec}$. The samples were again treated with 1 unit of uracilDNA glycosylase, heated, extracted with phenol and chloroform, and precipitated with ethanol. After drying, the samples were resuspended in $10 \mu$ l of the linear amplification mixture; however, 1 pmole of ${ }^{32}$ P-end-labeled LP1GT2A was used in place of the dUMP-containing primers. A final 100 thermal cycles were carried out with the profile used in the second round of linear amplification. These products were precipitated with 1 volume of isopropanol in the presence of $2 \mathrm{M}$ ammonium acetate and rinsed once with $70 \%$ ethanol. The samples were resuspended in $10 \mu \mathrm{l}$ of $1 \times$ rapid air thermocycler buffer, and $5 \mu$ l of loading dye was added. The amplification products were separated on $7 \%$ denaturing (urea/formamide) polyacrylamide gels, and autoradiography was carried out at $-90^{\circ} \mathrm{C}$ for $48 \mathrm{hr}$.

\section{RESULTS AND DISCUSSION}

Amplification of $d(C A)_{n}$ sequences by PCR often produces anomalous products. At one particular $\mathrm{d}(\mathrm{CA})_{\mathrm{n}}$ marker, LPL1GTR2, the mutant fragments make up $\sim 55 \%$ of the total PCR product following 30 thermal cycles, and the wildtype fragments contribute the remaining $45 \%$ (Fig. 1, lane 1). This proportion of mutant to wild-type fragments is independent of the annealing temperature $\left(55^{\circ} \mathrm{C}\right.$ or $\left.60^{\circ} \mathrm{C}\right)$ and the primer combination used in the PCR (data not shown). Assuming a constant mutation rate and no reversion to wild type, the fraction of wild-type molecules present following any given PCR cycle can be calculated using the following formula:

$$
W_{n}=\left(1-\frac{1}{2} \mu\right)^{n}
$$


where $W$ is the fraction of the wild-type product, $n$ is the cycle number, and $\mu$ is the mutation rate per PCR cycle. Solving for $\mu$ produces the following formula:

$$
\mu=2\left(1-W_{n}^{1 / n}\right)
$$

We calculate that a constant mutation rate of $\sim 5 \%$ per PCR cycle would be required to account for the final fractions of wild-type and mutant products. However, the mutation rate may not be constant throughout PCR; this will depend on the mechanism that generates the anomalous fragments. Mechanisms that are independent of template concentration, for example, polymerase slippage, would be expected to generate more uniform mutation rates, whereas recombinational mechanisms, which are dependent on template concentration, would produce rates that tend to increase during the course of the PCR. The template concentrations used in our test designed to detect anomalous fragments following a single round of primer extension simulate those found in the last few cycles of PCR. We therefore assume that under these conditions, the calculated $5 \%$ mutation rate is a minimum estimate of the true mutation rate. This assumption is strengthened further by the fact that we ignored the possibility of reversion to wild type. Reversions, which cannot be detected in these assays, would decrease the estimate of $\mu$ relative to the true mutation rate. Reversions are relatively uncommon occurrences during the PCR amplification of $d(C A)$ repeats (these sequences tend to contract during PCR); however, reversions undoubtedly occur. Figures 1 and 3 demonstrate that $\mathrm{d}(\mathrm{CA})$ repeats can occasionally expand and thus have the potential to revert to wild-type (note that the PCR products in each lane contain an anomalous fragment that is two nucleotides longer than the largest wild-type allele).

Our results indicate that a single round of primer extension in the presence of just one of the two PCR primers yields a mutation rate considerably less than the required $5 \%$ (Fig. 1, lane 2 ). Because this assay should detect all slippage products, this result suggests that slippage alone cannot account for all of the mutant products generated during the PCR amplification of d(CA) repeats. Recombinational mechanisms, for example, out-of-register annealing of trun- cated products in subsequent PCR cycles and out-of-register template-switching, may also be involved in the generation of the anomalous fragments. Linear amplification does not produce a significant ladder-like pattern (Fig. 1). However, no conclusions regarding recombinational mechanisms should be drawn from these results, because the template concentration used in the experiments was relatively low. Out-of-register switches to preexisting templates apparently cannot account for all of the mutant products, because these events would be indistinguishable from slippage and would have been detected during a single round of primer extension. However, these results do not address the possibility of out-of-register template switches between the complementary nascent strands that are being synthesized from the two primers in the PCR mixture. It remains possible that a combination of the mechanisms described here or others not discussed may be responsible for the generation of the anomalous PCR products.

The observation that linear amplification (i.e., multiple thermal cycles using a single primer) did not generate a significant ladder-like pattern (Fig. 1, lane 3) allowed us to develop experimental protocols designed to reduce anomalous amplification products. In general, these procedures rely on multiple rounds of linear amplification to achieve the necessary sensitivity for detection (Fig. 2). A single primer and numerous thermal cycles are used to linearly amplify a target $\mathrm{d}(\mathrm{CA})_{\mathrm{n}}$ marker. The primer is then eliminated, and the next round of linear amplification is performed with a second primer that anneals to the 3 ' ends of the extension products from the first round. The second primer is eliminated, and a third round of linear amplification is carried out with an end-labeled primer. Alternatively, one can use an end-labeled primer in the second round of linear amplification and omit the third round. We refer to these two techniques as three- and two-stage linear amplification (3-SLA and 2-SLA), respectively.

Figure 3 shows a comparison between PCR and 3-SLA amplification products of the LPL1GTR2 marker. Figure 3B illustrates the products obtained by PCR. Each allele is represented by several bands, which differ in length by two nucleotides from their nearest neighbors; this complex pattern makes genotypic interpretation more difficult. Figure $3 \mathrm{C}$ shows the amplification products generated by the 3-SLA technique. The banding patterns are much less complex and are easily interpreted. Figure 3 illustrates the difficulty that can be encountered when one attempts to determine by means of PCR whether an individual is homozygous or heterozygous. All six siblings are heterozygotes, each possessing alleles that differ by a single dinucleotide repeat. By following empirically derived guidelines and relying on the structure of the pedigree, the genotype for each individual can be determined by PCR; however, the 3-SLA technique allows for a more direct interpretation without relying on a set of guidelines or the family structure.

We used a PhosphorImager (Molecular Dynamics, Sunnyvale, CA) to quantitate the amount of wild-type and anomalous amplification products generated by 3-SLA and PCR techniques. The ratio of the fraction of anomalous products generated during PCR to the fraction of anomalous products generated during 3-SLA was determined; from this analysis we estimate that the 3-SLA technique offers a greater than fourfold reduction of anomalous $d(C A)_{n}$ amplification products when compared with PCR.

A critical step in the 2-SLA and 3-SLA procedures is the elimination of the primer from the previous round of linear amplification. If the primer is not eliminated, it can participate in the subsequent amplification process and generate the ladder-like pattern observed following PCR. An efficient method for eliminating unwanted primers incorporates both replacement of dTMPs with dUMPs during the chemical synthesis of the oligodeoxynucleotide and treatment of samples with uracil-DNA glycosylase (UDG) following each round of linear amplification. This enzyme hydrolyzes uracil-glycosidic bonds at all uracilDNA sites, creating alkali- or heat-sensitive apyrimidinic sites in the DNA strand. ${ }^{(11,12)}$ Treatment with UDG, followed by incubation at high temperature, destroys both the excess dUMPcontaining primers and the $5^{\prime}$ ends of their extension products. Thus, each successive round of linear amplification produces extension products that are shorter than the previous round. We designed our dUMP-containing primers such that the dUMPs were fairly evenly spaced throughout the primer except 


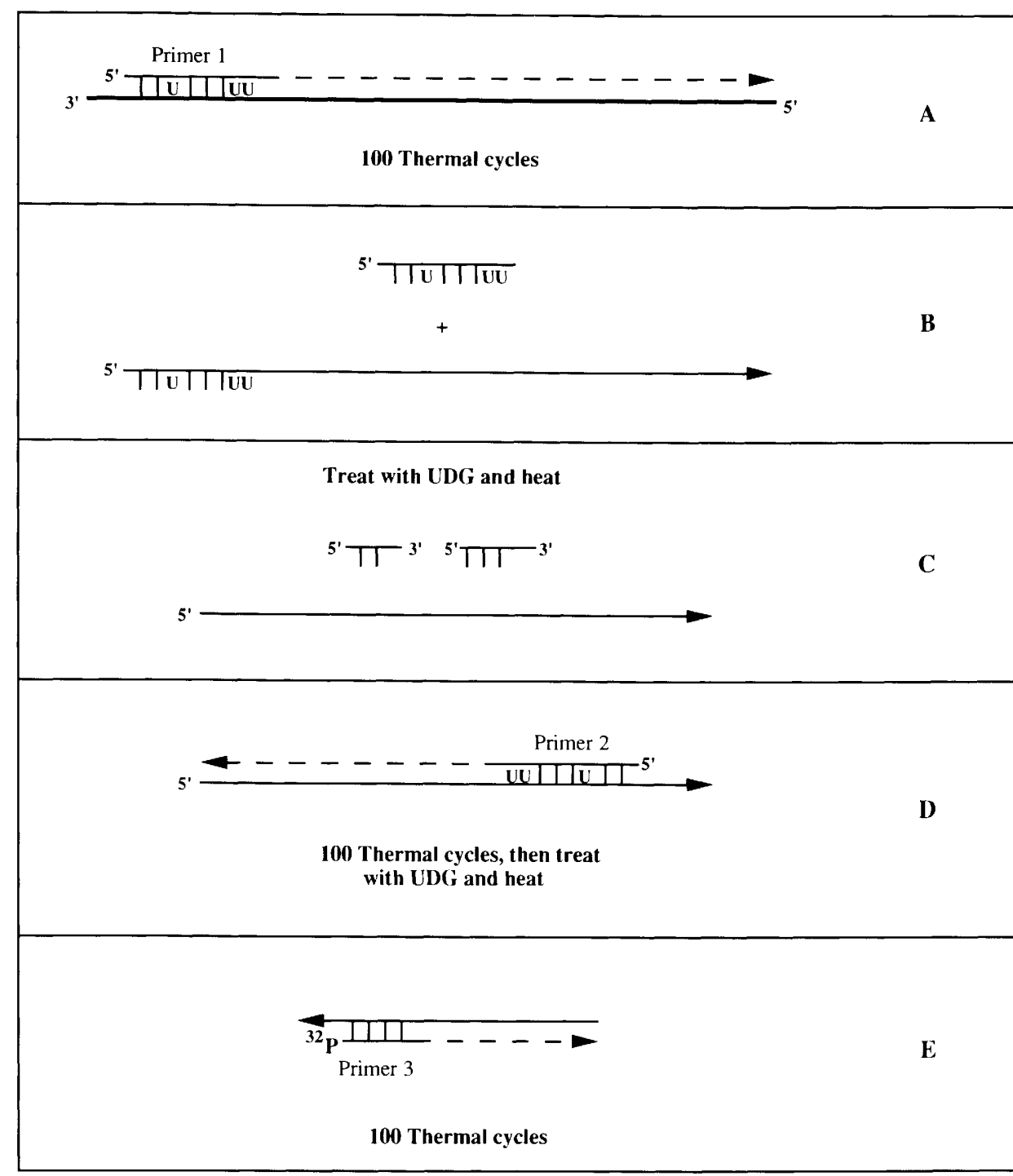

FIGURE 2 Schematic diagram of three-stage linear amplification. The solid line without an arrow represents the original template, whereas those with arrows represent DNA that has been synthesized previously during linear amplification. Broken lines with arrows represent DNA replication. $(A)$ One hundred thermal cycles are carried out using primer 1. This primer contains dUMPs in place of dTMPs and represents primer LPL1dU in the text. $(B)$ The components that are present after the first stage of linear amplification include excess primer 1 and extension products of primer 1. (C) Excess primer 1 and the $5^{\prime}$ end of its extension products are eliminated by treatment with UDG and heat. $(D)$ Primer 2, which anneals to the 3 ' end of the extension products from the first round of linear amplification, is added, and the samples are subjected to another round of 100 thermal cycles. This primer corresponds to primer RLPdU in the text. Excess primer 2 and the $5^{\prime}$ end of its extension products are eliminated. $(E){ }^{32} \mathrm{P}$-End-labeled primer 3 (primer LP1GT2A in the text) is added, and a final round of 100 thermal cycles is carried out.

that the $3^{\prime}$ end contained at least two adjacent dUMPs. This assured us that the primers would be cut into fragments containing no more than seven nucleotides - a size that would effectively prevent unwanted primer extensions.

Both 2-SLA and 3-SLA require numerous thermal cycles to achieve a visible signal by autoradiography, because they rely on linear amplification from a single primer during each thermal cycling reaction. This is in contrast to PCR, which exponentially amplifies a segment of DNA by synthesizing both strands of the double helix during each cycle. To achieve the required signal, it was necessary to perform 100 cycles of primer extension for each round of lin- ear amplification; thus, a very rapid thermal cycler was a practical requirement. The 1605 Air Thermo-Cycler meets this requirement; it is capable of performing a complete thermal cycle in $<30 \mathrm{sec}$. ${ }^{(13)}$ For this reason we performed all of the reactions in this study using the 1605 Air Thermo-Cycler.

Either 2-SLA or 3-SLA can be used to generate an accurate genotype of an individual at $d(C A)_{n}$ loci. The advantages of the 2-SLA technique include speed and simplicity; the major disadvantage is the requirement for a large amount (2 $\mu \mathrm{g}$ ) of starting genomic DNA, which may contribute to a high lane background (data not shown). The 3-SLA technique has the advantage of increased sensitivity and specificity. It is capable of generating a genotype from an initial $200 \mathrm{ng}$ of genomic DNA; in this respect, it is comparable to PCR. Furthermore, the use of three primers increases the specificity of the amplification. The disadvantages of 3-SLA include increased time and labor. The products from the first two rounds of linear amplification must be extracted with organic solvents and precipitated, presumably because the increased concentrations of glycerol or denatured protein lead to inhibition of Taq DNA polymerase.

LPL1GTR2 is located near the $5^{\prime}$ end of the lipoprotein lipase gene (S.J. Odelberg, R.B. Weiss, A. Hata, and R. White, in prep.). We have found that our primer set identifies a fairly common null allele, which can lead to apparent second-order parental exclusions when genotyping families. The specific molecular defect has not been ascertained but could reflect any one of a variety of mutations, including a single-base substitution at one of the two priming sites, a deletion at one of the priming sites, or even a larger deletion encompassing the entire amplicon.

We used 3-SLA to amplify a second $\mathrm{d}(\mathrm{CA})_{\mathbf{n}}$ sequence, LNS-CA, ${ }^{(14)}$ which also generates complex banding patterns upon amplification by PCR. This d(CA) repeat is part of the 3 '-untranslated region of the DP1 gene, which is located $30-70 \mathrm{~kb}$ downstream of the adenomatous polyposis coli $(A P C)$ gene. The 3-SLA technique produced readily interpretable genotypic patterns, demonstrating the general applicability of this procedure (data not shown).

It is not surprising that the 2-SLA and 3-SLA procedures reduce the amount of 


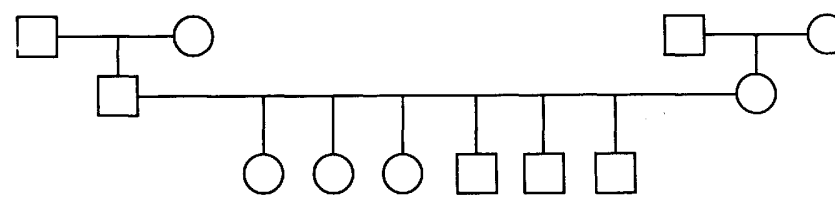

A

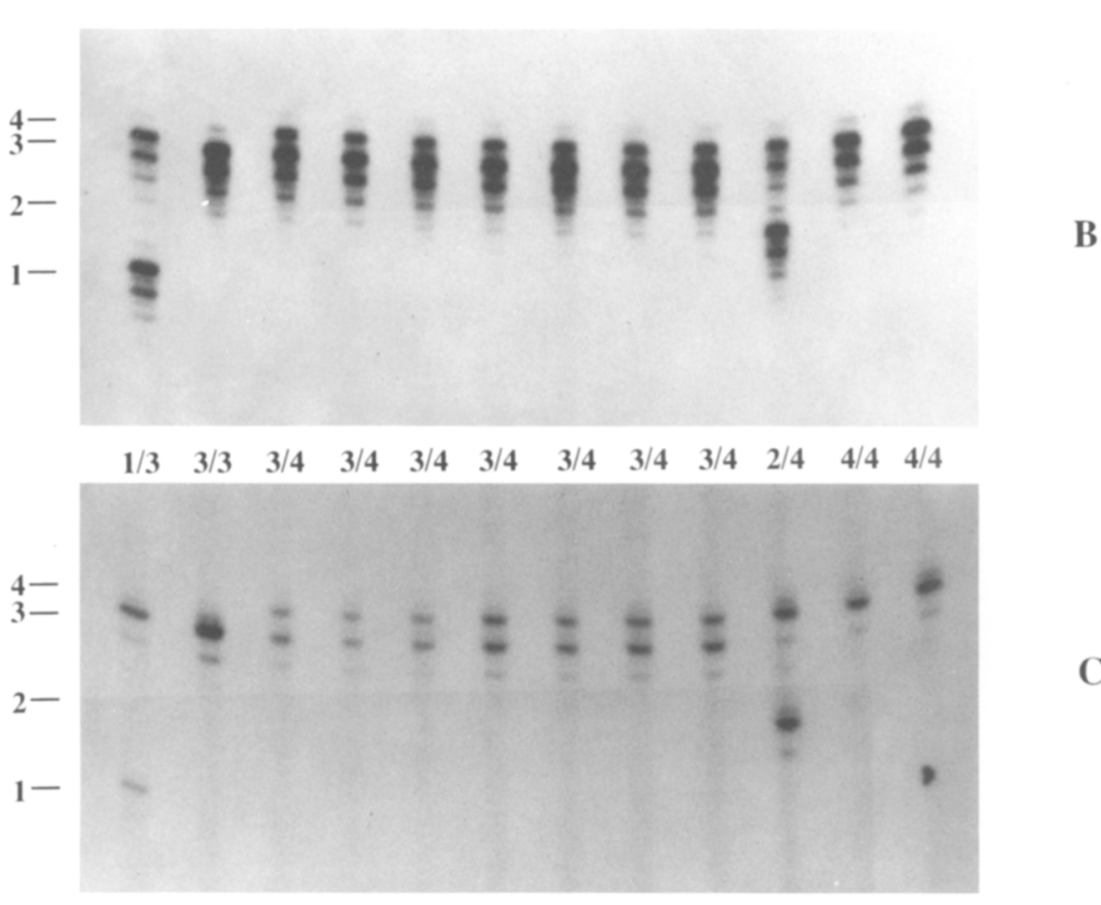

FIGURE 3 A direct comparison of the PCR and 3-SLA techniques. Amplification of the LPL1GTR2 marker. (A) A partial pedigree of kindred 1463 of the CEPH reference panel. The amplification products of each individual's DNA in $B$ and $C$ are lined up directly under their representative symbol in the pedigree. $(B)$ The PCR products for each DNA sample. $(C)$ The 3-SLA products for each DNA sample. Although some anomalous bands remain, they are greatly reduced in intensity and do not interfere with genotypic interpretation. (Note: The alleles are numbered from the smallest to the largest; these numbers appear at left.) The assigned genotype of each individual is listed.

anomalous fragments generated during amplification. Mutant extension products synthesized during linear amplification cannot be used as template during that series of primer extensions and therefore accumulate linearly. This is in contrast to PCR, where mutant extension products can participate as templates in subsequent cycles and be exponentially amplified. For this reason, the 2-SLA and 3-SLA procedures are expected to generate fewer anomalous fragments and a less complex banding pattern.

In conclusion, we have shown that 3-SLA is capable of amplifying a d(CA) sequence without generating a significant ladder-like pattern. This is in marked contrast to PCR, which often produces complex banding patterns. PCR-based analyses of $d(C A)_{n}$ markers have been valuable both in constructing genetic linkage maps ${ }^{(15,16)}$ and in dem- onstrating linkage to disease-causing genes. $^{(17-19)}$ Because the 3-SLA technique allows accurate genotypes to be assigned to each individual without relying on empirically derived guidelines and pedigree structures, it extends the utility of these hypervariable loci to investigations involving disputed parentage and individualization of biological specimens.

\section{ACKNOWLEDGMENTS}

We are grateful to Carl T. Wittwer for sharing his thermo-cycler with us during the initial stages of this investigation. We thank Josh Cherry for help in simplifying the mathematical formulae, Tami Elsner for computer programming assistance, and Ying Li for helpful suggestions. We also thank Ruth Foltz for editing the manuscript. This work was supported by National Institutes of Health grant R01HG00367. R.W. is an investigator for the Howard Hughes Medical Institute.

\section{REFERENCES}

1. Litt, M. and J.A. Luty. 1989. A hypervariable microsatellite revealed by in vitro amplification of a dinucleotide repeat within the cardiac muscle actin gene. Am. J. Hum. Genet. 44: 397-401.

2. Tautz, D. 1989. Hypervariability of simple sequences as a general source for polymorphic DNA markers. Nucleic Acids Res. 17: 6463-6471.

3. Miesfeld, R., M. Krystal, and N. Arnheim. 1981. A member of a new repeated sequence family which is conserved throughout eucaryotic evolution is found between human $\delta$ and $\beta$ globin genes. Nucleic Acids Res. 9: 5931-5947.

4. Hamada, H., M.G. Petrino, and T. Kakunaga. 1982. A novel repeated element with Z-DNA-forming potential is widely found in evolutionary diverse eukaryotic genomes. Proc. Natl. Acad. Sci. 79: 6465-6469.

5. Weber, J.L. and P.E. May. 1989. Abundant class of human DNA polymorphisms which can be typed using the polymerase chain reaction. Am. J. Hum. Genet. 44: 388-396.

6. Saiki, R.K., D.H. Gelfand, S. Stoffel, S.J. Scharf, R. Higuchi, G.T. Horn, K.B. Mullis, and H.A. Erlich. 1988. Primer-directed enzymatic amplification of DNA with a thermostable DNA polymerase. Science 239: 487-491.

7. Pääbo, S., D.M. Irwin, and A.C. Wilson. 1990. DNA damage promotes jumping between templates during enzymatic amplification. J. Biol. Chem. 265: 4718-4721.

8. Meyerhans, A., J.-P. Vartanian, and S. Wain-Hobson. 1990. DNA recombination during PCR. Nucleic Acids Res. 18: 16871691.

9. Jansen, R. and F.D. Ledley. 1990. Disruption of phase during PCR amplification and cloning of heterozygous target sequences. Nucleic Acids Res. 18: 51535156.

10. Marton, A., L. Delbecchi, and P. Bourgaux. 1991. DNA nicking favors PCR recombination. Nucleic Acids Res. 19: 24232426.

11. Stuart, G.R. and R.W. Chambers. 1987 Synthesis and properties of oligodeoxynucleotides with an AP site at a preselected position. Nucleic Acids Res. 15: 7451-7462.

12. Longo, M.C., M.S. Berninger, and J.L. Hartley. 1990. Use of uracil DNA glycosylase to control carry-over contamination in polymerase chain reactions. Gene 93: $125-128$. 
13. Wittwer, C.T., G.C. Fillmore, and D.J. Garling. 1990. Minimizing the time required for DNA amplification by efficient heat transfer to small samples. Anal. Biochem. 186: 328-331.

14. Spirio, L., G. Joslyn, L. Nelson, M. Leppert, and R. White. 1991. A CA repeat 30$70 \mathrm{~kb}$ downstream from the adenomatous polyposis coli (APC) gene. Nucleic Acids Res. 19: 6348.

15. NIH/CEPH Collaborative Mapping Group. 1992. A comprehensive genetic linkage map of the human genome. Science 258: 67-86.

16. Weissenbach, J., G. Gyapay, C. Dib, A. Vignal, J. Morissette, P. Millasseau, G. Vaysseix, and M. Lathrop. 1992. A secondgeneration linkage map of the human genome. Nature 359: 794-801.

17. Wijmenga, C., R.R. Frants, O.F. Brouwer, P. Moerer, J.L. Weber, and G.W. Padberg. 1990. Location of facioscapulohumeral muscular dystrophy gene on chromosome 4. Lancet 336: 651-653.

18. Hilbert, P., K. Lindpaintner, J.S. Beckmann, T. Serikawa, F. Soubrier, C. Dubay, P. Cartwright, B. De Gouyon, C. Julier, S. Takahasi, M. Vincent, D. Ganten, M. Georges, and G.M. Lathrop. 1991. Chromosomal mapping of two genetic loci associated with blood-pressure regulation in hereditary hypertensive rats. Nature 353: 521-529.

19. Jacob, H.J., K. Lindpaintner, S. Lincoln, K. Kusumi, R.K. Bunker, Y.-P. Mao, D. Ganten, V.J. Dzau, and E.S. Lander. 1991. Genetic mapping of a gene causing hypertension in the stroke-prone spontaneously hypertensive rat. Cell 67: 213-224.

Received February 23, 1993; accepted in revised form May 10, 1993. 


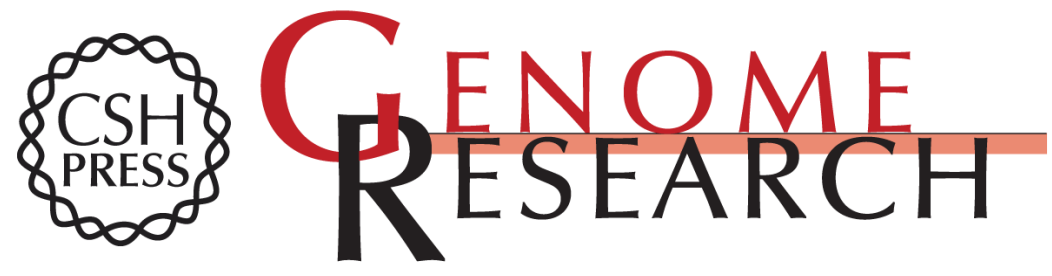

\section{A method for accurate amplification of polymorphic CA-repeat sequences.}

S J Odelberg and R White

Genome Res. 1993 3: 7-12

References This article cites 19 articles, 4 of which can be accessed free at: http://genome.cshlp.org/content/3/1/7.full.html\#ref-list-1

\section{License}

Email Alerting

Receive free email alerts when new articles cite this article - sign up in the box at the Service top right corner of the article or click here.

\section{Affordable, Accurate Sequencing.}

To subscribe to Genome Research go to: https://genome.cshlp.org/subscriptions 